\title{
ANALYTICAL SOLUTIONS FOR EXPANDING FIREBALLS
}

\author{
Chao Li and Re'em SARI \\ Theoretical Astrophysics, California Institute of Technology, MC 130-33, Pasadena, CA 91125 \\ Received 2007 January 16; accepted 2007 December 9
}

\begin{abstract}
Many models of gamma-ray bursts (GRBs) as well as of soft gamma repeaters (SGRs) involve a fireball — an optically thick concentration of radiation energy with a high ratio of energy density to rest mass. We study the asymptotic behavior of an ultrarelativistic fireball consisting of electron-positron pairs and photons. We show that in the ultrarelativistic limit, after photons decouple from the pairs, the photon distribution function remains a blackbody spectrum in some appropriate Lorentz frame, allowing us to define an effective Lorentz factor and temperature for the photon gas. We also study the freezing out of electron-positron pairs and their asymptotic Lorentz factor $\gamma_{\infty}$. The dependence of these quantities on initial conditions can be described by simple scaling laws. We apply our results to SGR 1806-20 and find that the energy carried by electron-positron pairs is higher than calculated by former estimates, but is still an order of magnitude short of the minimum energy required to produce the observed afterglow. A viable solution of the energy budget is that the fireball is loaded by baryons or electromagnetic flux.
\end{abstract}

Subject headings: gamma rays: bursts — hydrodynamics — radiative transfer

\section{INTRODUCTION}

The release of a large amount of energy into a compact region can lead to an opaque electron-positron-photon fireball. The formation and evolution of fireballs are of interest in astrophysics, especially for the understanding of gamma-ray bursts (GRBs) at cosmological distances (Goodman 1986; Paczynski 1986; Shemi \& Piran 1990; Piran et al. 1993; Mészáros et al. 1993) and soft gamma repeaters (SGRs; Kouveliotou et al. 1998; Thompson \& Duncan 1995, 1996). The evolution of an electron-positron-photon fireball consists of several phases (Shemi \& Piran 1990; Grimsrud $\&$ Wasserman 1998). Initially, the electrons, positrons, and photons are in thermal equilibrium due to high temperature and large optical depth. As the fireball expands, its temperature decreases, and the equilibrium number density of electrons and positrons decreases. The optical depth for pair annihilation drops below unity, and their density deviates from thermal equilibrium below a temperature of about $20 \mathrm{keV}$. Around the same time, the optical depth for photon scattering drops below unity so that photons decouple from pairs and begin free-streaming. Further expansion causes the density of electron-positron pairs to decrease, but their total number is now conserved. The Lorentz factor of electrons increases until the optical depth for scattering off photons becomes small. Then the pairs decouple from the photons and, therefore, no longer accelerate. If we consider the fireball as a steady state wind, there will be a constant number of pairs per unit time $\dot{N}_{\infty}$ arriving at infinity with Lorentz factor $\gamma_{\infty}$.

In this paper we analytically study these fireball processes. We assume a steady state wind and focus on deriving exact solutions for $\dot{N}_{\infty}$ and $\gamma_{\infty}$. These quantities have only been estimated to an order of magnitude previously (Grimsrud \& Wasserman 1998; Nakar et al. 2005). We prove in $\S 2$ that after photons decouple from the pairs, the photons remain thermal in a Lorentz frame that is accelerating as $\gamma_{\mathrm{ph}} \propto r$. We define the Lorentz factor of this frame, $\gamma_{\mathrm{ph}}$, as the Lorentz factor of the photons. When the fireball is optically thick, the pair Lorentz factor $\gamma_{e}$ is almost equal to that of the photons $\gamma_{\mathrm{ph}}$, since the photons are dragging the pairs. As the fireball expands and becomes sufficiently optically thin, $\gamma_{\mathrm{ph}}$ and $\gamma_{e}$ start to deviate from each other, with $\gamma_{e}<\gamma_{\mathrm{ph}}$. Finally, $\gamma_{e}$ approaches its coasting value $\gamma_{\infty}$.
In $\S 3$ we calculate the number of pairs per unit time that survive or freeze out once the annihilation timescale becomes longer than the dynamical time. We arrive at an exact expression under the assumption that the pair Lorentz factor during freezeout is very similar to that of the photons and that the temperature at freezeout, $k_{\mathrm{B}} T_{ \pm}$, is significantly below the electron rest mass. In $\S 4$ we derive an expression for the coasting Lorentz factor. We generalized our results in $\S 5$ for the case that the fireball is loaded with some amount of baryons and their associated electrons, or with magnetic fields. We discuss the applications to SGRs in $\S 6$.

\section{FREELY STREAMING PHOTONS}

The sudden release of a large amount of energy in a compact region produces an expanding fireball. Relativistic dynamics in spherical geometry ensures that the matter expands such that its Lorentz factor is proportional to the radius (Shemi \& Piran 1990; Piran et al. 1993). Before the photons reach the photosphere, they are in thermal equilibrium, and the distribution function is of blackbody form. If at some radius the photons begin to stream freely without scattering, then, as we will show below, photons remain thermalized as long as we observe them in a Lorentz frame that continues to accelerate linearly with distance, $\gamma_{\mathrm{ph}} \propto r$. As a side note, we mention that since this relation is true for no scattering, $\gamma_{\mathrm{ph}}$ continues to increase all the way to the observer. For example, in the giant flare of SGR 1806-20 the observed $200 \mathrm{keV}$ photons, when they reach the observer, have a thermal distribution with a temperature of $4 \times 10^{-12} \mathrm{eV}$ if viewed in a frame that has a Lorentz factor $\gamma_{\mathrm{ph}}=5 \times 10^{16}$.

In Figure 1, photons emitted from point 1 on the photosphere arrive at point 2 , and $\theta_{10}$ and $\theta_{20}$ are angles measured in the observer frame. Correspondingly, $\theta_{1}$ and $\theta_{2}$ denote the same angles measured in the comoving frames of the photon gas at points 1 and 2 . When the fireball reaches the photosphere, $\gamma_{\mathrm{ph}} \gg 1$, which means that the transverse optical depth is of the order $\gamma_{\mathrm{ph}}^{2} \gg 1$; thus, point 2 can only receive tiny amount of photons which are emitted from high-latitude regions on the photosphere. Therefore, it is reasonable to assume

$$
\theta \ll 1, \quad \theta_{10} \ll 1, \quad \theta_{20} \ll 1 .
$$




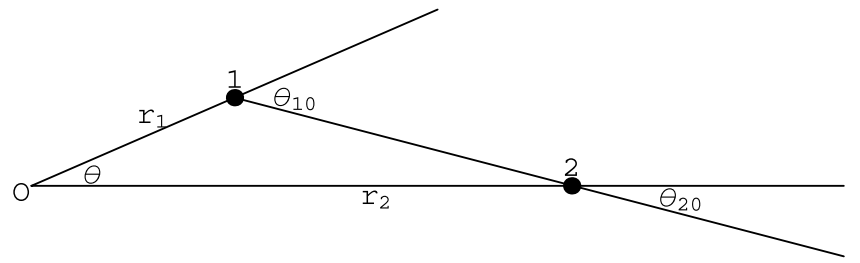

FIG. 1.-Photon emitted at point 1 is received at point 2. Point $\mathrm{O}$ is the origin of the fireball, point 1 lies on the photosphere, and point 2 is an arbitrary point outside the photosphere. All the angles are exaggerated since $\theta, \theta_{10}, \theta_{20} \ll 1$.

We note that although $\theta_{10}$ and $\theta_{20}$ are very small, their counterparts $\theta_{1}$ and $\theta_{2}$ in the comoving frames can be large. From Liouville's theorem, the phase-space distribution function is conserved along each collisionless trajectory. Thus, we get the relation for the photon distribution function,

$$
f\left(\nu_{2}, \theta_{2}\right)=f\left(\nu_{20}, \theta_{20}\right)=f\left(\nu_{10}, \theta_{10}\right)=f\left(\nu_{1}, \theta_{1}\right) .
$$

Here the second equality is from Liouville's theorem and the first and third equalities are from the Lorentz invariance of the phasespace distribution function. Since we assume the photon distribution at point 1 is that of a blackbody in its comoving frame, we obtain

$$
f\left(\nu_{2}, \theta_{2}\right)=f\left(\nu_{1}, \theta_{1}\right)=\frac{1}{e^{h \nu_{1} / k_{\mathrm{B}} T_{1}}-1} .
$$

In order to express the photon distribution function at point 2 in terms of photon frequency $\nu_{2}$ and angle $\theta_{2}$, we need to do Lorentz transformations at points 1 and 2 and use the relation $\nu_{10}=\nu_{20}$ for collisionless photons. We obtain

$$
\frac{\nu_{1}}{T_{1}}=\frac{\nu_{2} \gamma_{\mathrm{ph}, 2}}{T_{1} \gamma_{\mathrm{ph}, 1}}\left(1+\beta_{2} \cos \theta_{2}\right) \gamma_{\mathrm{ph}, 1}^{2}\left(1-\beta_{1} \cos \theta_{10}\right) \cong \frac{\nu_{2} \gamma_{\mathrm{ph}, 2}}{T_{1} \gamma_{\mathrm{ph}, 1}}
$$

where $\gamma_{\mathrm{ph}, 1}$ and $\gamma_{\mathrm{ph}, 2}$ denote corresponding photon Lorentz factors at points 1 and 2 . In the above argument, we use equation (1), $r_{1} / \gamma_{\mathrm{ph}, 1}=r_{2} / \gamma_{\mathrm{ph}, 2}$, and a geometrical relation $r_{1} \theta_{10}=r_{2} \theta_{20}$. Therefore, the photon distribution function at point 2 is

$$
f\left(\nu_{2}, \theta_{2}\right)=\frac{1}{e^{h \nu_{1} / k_{\mathrm{B}} T_{1}}-1}=\frac{1}{e^{h \nu_{2} /\left(k_{\mathrm{B}} T_{1} \gamma_{\mathrm{ph}, 1} / \gamma_{\mathrm{ph}, 2}\right)}-1} .
$$

Therefore, by defining the photon Lorentz factor $\gamma_{\mathrm{ph}}$ to be proportional to $r$, the photon distribution function remains a blackbody spectrum in this comoving frame, with the corresponding temperature inversely proportional to $r$. As such, the distribution function is isotropic, $f\left(\nu_{2}, \theta_{2}\right)=f\left(\nu_{2}\right)$. In other words, if we observe the freely streaming photons in a carefully chosen Lorentz frame, we will always observe an isotropic blackbody spectrum. In deriving this conclusion, we have only used the following properties of free-streaming photons: $\gamma_{\mathrm{ph}} \propto r$ and $\theta_{10} \ll 1$. The result is therefore quite general.

Clearly, the free-streaming of photons is a valid assumption only when the optical depth for scattering is well below unity. It is therefore important to explain why those conclusions continue to hold even when the optical depth is around unity. The collision effect vanishes as long as the electron-positron pairs are thermal. It is the photons that keep the electrons thermal as the fireball expands. However, at the photosphere, where the photons escape and the optical depth for scattering of a photon off an electron is of order unity, the density of photons far exceeds that of pairs. An order-of-magnitude estimate gives the photon-to-pair number ratio to be

$$
e^{\left(m_{e} c^{2}\right) / k_{\mathrm{B}} T} \sim e^{(511) / 20} \sim 10^{11},
$$

where we have substituted the temperature $T \sim 20 \mathrm{keV}$ at the photosphere, which will be explained below in $\S 3$. Therefore, the last few collisions that a photon experiences are with electrons that have already been scattered by many photons. Because of the rest mass of the pairs, the criterion for the electrons to stay thermal is that the photon-to-pair number ratio exceeds $m_{e} c^{2} /$ $k_{\mathrm{B}} T \sim 20$, which is easily satisfied.

The observed flux per energy interval as seen by a stationary observer is

$$
\frac{d F}{d \nu}=\frac{4 \pi h \nu^{3}}{c^{2}} \int_{-1}^{1} \frac{\mu d \mu}{\exp \left[\gamma h \nu(1-\beta \mu) / k_{\mathrm{B}} T\right]-1},
$$

where $\gamma$ and $T$ are the Lorentz factor and temperature of the photon gas at the observer, respectively. Similar expressions for this spectrum were obtained by Goodman (1986) and Grimsrud $\&$ Wasserman (1998). In the limit of large $\gamma$, this can be reduced to

$$
\frac{d F}{d \nu}=\frac{4 \pi \nu^{2} k_{\mathrm{B}} T}{c^{2} \gamma} \ln \left(\frac{2 \gamma k_{\mathrm{B}} T}{h \nu}\right)
$$

at low energies and

$$
\frac{d F}{d \nu}=\frac{4 \pi \nu^{2} k_{\mathrm{B}} T}{c^{2} \gamma} \exp \left(\frac{-h \nu}{2 \gamma k_{\mathrm{B}} T}\right)
$$

at high energies. Compared to a blackbody, the peak of the flux is broader, and the slope at low energies is slightly shallower. Note that the product $\gamma T$ appearing in the exponent and in the logarithm is independent of distance, while the ratio $T / \gamma$ appearing in the prefactors is decreasing as the distance squared, as expected.

\section{FREEZEOUT OF PAIRS}

In this section we assume that the fireball has a constant luminosity (steady wind) and consider the freezeout of electronpositron pairs. This assumption is valid if the variability timescale is longer that the light crossing time at the source. Initially the fireball is hot. The electron-positron pairs and photons are in thermal equilibrium with each other. Therefore, for a given temperature the density of pairs is completely determined. Near the photosphere, photons decouple from the pairs. But due to the large ratio between the numbers of photons and pairs (eq. [6]), pairs are still accelerated efficiently by photons. On the other hand, because the local expansion rate is larger than the pair annihilation rate, the pair annihilation process gradually stops and the number of pairs emitted per unit time freezes to some limiting value $\dot{N}_{\infty}$. Therefore, in this section we assume $\gamma_{e}=$ $\gamma_{\text {ph }} \propto r$ when calculating $\dot{N}_{\infty}$. We define the effective initial radius of the fireball by $r_{i} \equiv r / \gamma_{\mathrm{ph}}$. This definition allows $r_{i}$ to be somewhat different from the actual radius where the energy is released (e.g., the radius of a neutron star).

The equation describing the evolution of the number of pairs is (Grimsrud \& Wasserman 1998)

$$
\begin{array}{r}
\frac{d \dot{N}}{d r}=-\frac{r_{i}^{2}\left\langle\sigma_{\mathrm{ann}} v\right\rangle}{4 \pi r^{4} c^{2}}\left(\dot{N}^{2}-\dot{N}_{e, \mathrm{eq}}^{2}\right), \\
\dot{N}_{e, \text { eq }}=\frac{8 \pi c r^{3}}{h^{3} r_{i}}\left(2 \pi m_{e} k_{\mathrm{B}} T\right)^{3 / 2} e^{-m_{e} c^{2} / k_{\mathrm{B}} T},
\end{array}
$$


where $\dot{N} \equiv 4 \pi r^{2} n_{e} \gamma_{e} c$ is the number of pairs emitted per unit time and $\left\langle\sigma_{\mathrm{ann}} v\right\rangle$ is the pair annihilation cooling rate, which is almost constant for $k_{\mathrm{B}} T \ll m_{e} c^{2}$ (Svensson 1982). Throughout the paper, we assume $k_{\mathrm{B}} T \ll m_{e} c^{2}$ and adopt the cgs units. When the temperature is high enough, electron-positron pairs are in thermal equilibrium with photons. With the expansion and cooling of the fireball, the equilibrium is broken at some temperature $T_{ \pm}$, and the number of pairs freezes afterward.

We start with a rough estimate of the condition when the equilibrium breaks,

$$
\frac{d \dot{N}}{d r} \sim \frac{\dot{N}}{r} \sim \frac{r_{i}^{2}\left\langle\sigma_{\mathrm{ann}} v\right\rangle}{4 \pi r^{4} c^{2}} \dot{N}^{2} \sim \frac{r_{i}^{2}\left\langle\sigma_{\mathrm{ann}} v\right\rangle}{4 \pi r^{4} c^{2}} \dot{N}_{e, \mathrm{eq}}^{2}
$$

As an order-of-magnitude estimation, the total number of pairs at the equilibrium breaking point is

$$
\dot{N}_{ \pm} \sim \frac{4 \pi c^{2}}{r_{i}^{2}} \frac{r_{ \pm}^{3}}{\left\langle\sigma_{\text {ann }} v\right\rangle} .
$$

The corresponding temperature $T_{ \pm}$is determined by $\left(\dot{N}_{ \pm} \sim \dot{N}_{e, \text { eq }}\right)$

$$
\frac{c}{r_{i}\left\langle\sigma_{\mathrm{ann}} v\right\rangle\left(2 \pi m_{e} k_{\mathrm{B}} T_{ \pm}\right)^{3 / 2}}=\frac{2}{h^{3}} e^{-m_{e} c^{2} / k_{\mathrm{B}} T_{ \pm}} .
$$

Equation (13) serves as our definition of $T_{ \pm}$, the temperature at which pair annihilation is no longer effective. The right-hand side has an exponential dependence which is highly sensitive to $T_{ \pm}$, while the left-hand side depends only weakly on $T_{ \pm}$. Therefore, we can solve this equation iteratively. We start with $T_{0}=m_{e} c^{2} / k_{\mathrm{B}}$. Assume that at step $n$ we have obtained an approximate solution $T_{ \pm} \approx T_{n}$; then at step $n+1$ we can substitute $T_{n}$ to the left-hand side of equation (13) and solve for $T_{n+1}$. This iterative method converges very fast. We have tried some typical values of initial conditions and found that, after two iterations, $T_{2}$ is already very close to the numerical values of $T_{ \pm}$. Elegant analytical expressions for $T_{1}$ and $T_{2}$ can be written in terms of the dimensionless quantity $x \equiv(2 \pi)^{1 / 2} \alpha^{2}\left(r_{i} / \lambda_{e}\right)$, where $\alpha=1 / 137$ is the fine-structure constant and $\lambda_{e}$ is the Compton wavelength of the electron,

$$
\begin{gathered}
T_{1}=\frac{m_{e} c^{2} / k_{\mathrm{B}}}{\ln x}, \\
T_{ \pm} \approx T_{2}=\frac{m_{e} c^{2} / k_{\mathrm{B}}}{\ln x-(3 / 2) \ln \ln x} .
\end{gathered}
$$

Figure 2 shows $T_{1}$ and $T_{2}$ as functions of $r_{i}$ as well as the exact solution to equation (13). For $r_{i}>10^{4} \mathrm{~cm}$, the analytical expression $T_{2}$ is accurate to within $2 \%$.

It is easy to relate $T_{ \pm}$to the temperature at the photosphere. There are two differences here. First, the average velocity times the cross section in Thomson scattering is larger than that in pair annihilation, since $\left\langle\sigma_{\mathrm{ann}} v\right\rangle=3 \sigma_{\mathrm{T}} \mathrm{c} / 8$. Furthermore, there are twice as many pairs that are involved in Thomson scattering. These two reasons lead to a lower temperature than $T_{ \pm}$, which is also given by equation (14) but with $x=(16 / 3)(2 \pi)^{1 / 2} \alpha^{2}\left(r_{i} / \lambda_{e}\right)$; typical values are around $20 \mathrm{keV}$, which we used in $\S 2$.

Based on these estimates, we introduce the following dimensionless quantities

$$
\dot{N}=\frac{4 \pi r_{i} c^{2}}{\left\langle\sigma_{\text {ann }} v\right\rangle}\left(\frac{T_{i}}{T_{ \pm}}\right)^{3} \mathcal{N}, \quad r=\frac{T_{i} r_{i}}{T_{ \pm}} \mathcal{R}
$$

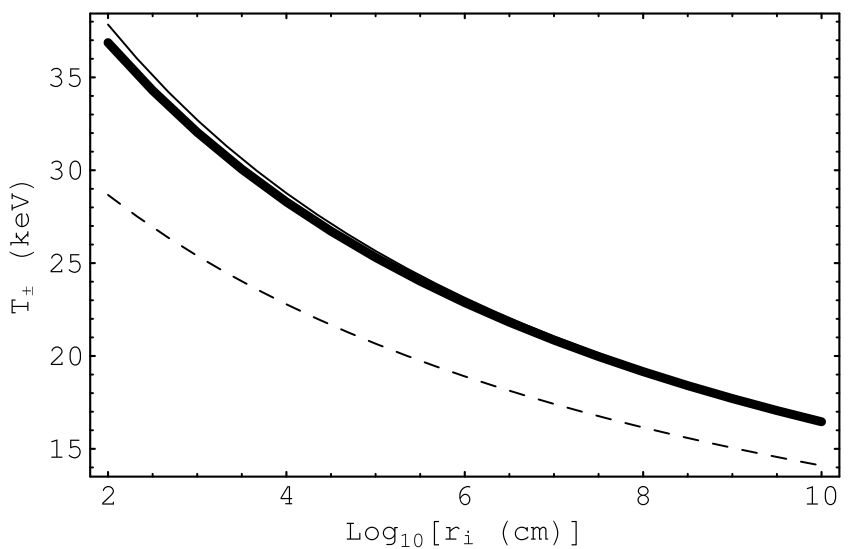

FIG. 2.-Comparison between the numerically exact solution of eq. (13) and our analytical approximation eq. (14). The horizontal axis is the logarithm of initial distance $r_{i}$ in units of centimeters. Dashed line, Our first approximation $T_{1}$ in eq. (14), solid line, our second approximation $T_{2}$ in eq. (14), thick solid line, numerical solution of eq. (13).

and equation (10) is converted into a dimensionless form,

$$
\frac{d \mathcal{N}}{d \mathcal{R}}=-\frac{\mathcal{N}^{2}}{\mathcal{R}^{4}}+\frac{1}{\mathcal{R}} e^{-\left[2 m_{e} c^{2}(\mathcal{R}-1)\right] /\left(k_{\mathrm{B}} T_{ \pm}\right)} .
$$

We are ultimately interested in

$$
\mathcal{N}_{\infty}\left(T_{ \pm}\right) \equiv \lim _{\mathcal{R} \rightarrow \infty} \mathcal{N}\left(\mathcal{T}_{ \pm}, \mathcal{R}\right)
$$

since this provides the flux of pairs arriving at infinity. The solution of equation (16) depends weakly on $T_{ \pm}$if $k_{\mathrm{B}} T_{ \pm} \ll m_{e} c^{2}$. In the Appendix, we have derived an approximate analytical expression

$$
\mathcal{N}_{\infty}\left(T_{ \pm}\right)=\frac{3}{1+\left(\ln 8-3 \gamma_{\text {Eu }}\right) T_{ \pm}-3 T_{ \pm} \ln T_{ \pm}},
$$

where $\gamma_{\mathrm{Eu}}=0.577216 \ldots$ is the Euler constant. Numerical integration of equation (16) results in $\mathcal{N}_{\infty}\left(T_{ \pm}=23 \mathrm{keV}\right)=1.95$, while the approximate equation (18) gives $\mathcal{N}_{\infty}\left(T_{ \pm}=23 \mathrm{keV}\right)=$ 2.09. Here $T_{ \pm}=23 \mathrm{keV}$ corresponds to $r_{i}=10^{6} \mathrm{~cm}$. From equation (15), we then obtain the number of pairs per unit time arriving at infinity,

$$
\dot{N}_{\infty} \approx 1.95 \frac{4 \pi r_{i} c^{2}}{\left\langle\sigma_{\mathrm{ann}} \nu\right\rangle}\left(\frac{T_{i}}{T_{ \pm}}\right)^{3} .
$$

For more general $T_{ \pm}$, we use equation (18) to obtain

$$
\dot{N}_{\infty} \approx \frac{3}{1+\left(\ln 8-3 \gamma_{\mathrm{Eu}}\right) T_{ \pm}-3 T_{ \pm} \ln T_{ \pm}} \frac{4 \pi r_{i} c^{2}}{\left\langle\sigma_{\mathrm{ann}} v\right\rangle}\left(\frac{T_{i}}{T_{ \pm}}\right)^{3} .
$$

In Figure 5, we plot the fractional error of the analytical expression to the numerical solution. From this comparison, we see that the analytical expression equation (20) is an accurate expression for the number of pairs arriving at infinity per unit time.

\section{COASTING OF PAIRS}

Even after the photons decouple from the pairs, the pairs are still accelerated by the free-streaming photons due to the large ratio between the numbers of photons and pairs. The consequence is that the Lorentz factor for pairs continues to grow. At large radii, 
the acceleration gradually stops, and the pairs enter their coasting phase. In this section we calculate the coasting Lorentz factor $\gamma_{\infty}$. The equation governing the evolution of the electron Lorentz factor is (Grimsrud \& Wasserman 1998)

$$
\frac{d \gamma_{e}}{d r}=\frac{\sigma_{\mathrm{tot}} F_{0}}{m_{e} c^{3}}
$$

where $\sigma_{\text {tot }}=\sigma_{a}+\sigma_{s}$ is the total cross section for both absorption and scattering and $F_{0}$ is the energy flux of photons in the rest frame of pairs. Since we have already proven that the photon distribution function is a blackbody in the local rest frame of freestreaming photons, we can do a simple Lorentz transformation to get the energy flux in the rest frame of electrons. This is different from the method of Grimsrud \& Wasserman (1998), who calculate a multipole expansion of the photon distribution function and only retain up to quadrupole terms. The relative Lorentz factor of the photon rest frame with respect to the pair rest frame is

$$
\gamma \cong \frac{1}{2}\left(\frac{\gamma_{\mathrm{ph}}}{\gamma_{e}}+\frac{\gamma_{e}}{\gamma_{\mathrm{ph}}}\right)
$$

So we get the energy flux $F_{0}$, and equation (21) is converted into

$$
\frac{d \gamma_{e}}{d r}=\frac{a T_{\mathrm{ph}}^{4}}{3}\left[\left(\frac{\gamma_{\mathrm{ph}}}{\gamma_{e}}\right)^{2}-\left(\frac{\gamma_{e}}{\gamma_{\mathrm{ph}}}\right)^{2}\right] \frac{\sigma_{\mathrm{tot}}}{m_{e} c^{2}},
$$

where $T_{\mathrm{ph}}=T_{i} r_{i} / r$ and $\gamma_{\mathrm{ph}}=r / r_{i}$ are the photon temperature and Lorentz factor, respectively, and the radiation constant $a=$ $8 \pi^{5} k_{\mathrm{B}}^{4} / 15 h^{3} c^{3}$. Using the transformation

$$
\Gamma=\gamma_{e}\left(\frac{3 m_{e} c^{2}}{r_{i} a T_{i}^{4} \sigma_{\mathrm{tot}}}\right)^{1 / 4}, \quad R=r\left(\frac{3 m_{e} c^{2}}{r_{i} a T_{i}^{4} \sigma_{\mathrm{tot}}}\right)^{1 / 4}
$$

the equation governing the acceleration of electrons can be written in dimensionless form as

$$
\frac{d \Gamma}{d R}=\frac{1}{R^{4}}\left[\left(\frac{R}{\Gamma}\right)^{2}-\left(\frac{\Gamma}{R}\right)^{2}\right] .
$$

Numerical integration of this dimensionless equation shows that the asymptotic limit is $\Gamma(R \rightarrow \infty) \approx 1.46$. Thus, the coasting Lorentz factor is

$$
\gamma_{\infty} \approx 1.46\left(\frac{8 \pi^{5} r_{i} T_{i}^{4} k_{\mathrm{B}}^{4} \sigma_{\mathrm{tot}}}{45 m_{e} h^{3} c^{5}}\right)^{1 / 4}
$$

The kinetic energy flux arriving at infinity is given by $L_{ \pm, \infty}=$ $2 \gamma_{\infty} m_{e} c^{2} \dot{N}_{\infty}$ or, since the photon luminosity is given by $L=$ $(16 \pi / 3) c r_{i}^{2} a T_{i}^{4}$,

$$
\begin{aligned}
L_{ \pm, \infty} & =11.6\left(\frac{k_{\mathrm{B}}^{4} m_{e}^{3} c^{7}}{h^{3} a^{4}}\right)^{1 / 4} \frac{\sigma_{\text {tot }}^{1 / 4}}{\left\langle\sigma_{\mathrm{ann}} v\right\rangle} \frac{L}{T_{ \pm}^{3} r_{i}^{3 / 4}} \\
& =0.19 \frac{m_{e}^{3 / 4} h^{9 / 4} c^{15 / 4} L}{\sigma_{\mathrm{T}}^{3 / 4}\left(k_{\mathrm{B}} T_{ \pm}\right)^{3} r_{i}^{3 / 4}}, \\
\gamma_{\infty} & =\gamma_{\infty, \text { pure }} \equiv 0.237\left(\frac{k_{\mathrm{B}}^{4}}{m_{e} h^{3} c^{9} a^{4}}\right)^{1 / 4} \frac{\sigma_{\text {tot }}^{1 / 4} L}{T_{i}^{3} r_{i}^{7 / 4}} \\
& =0.549\left(\frac{\sigma_{\mathrm{T}} L}{m_{e} c^{3} r_{i}}\right)^{1 / 4}
\end{aligned}
$$

where in the last equalities we have substituted $\sigma_{\text {tot }} \sim \sigma_{\mathrm{T}}$ and $\left\langle\sigma_{\mathrm{ann}} v\right\rangle \sim 3 \sigma_{\mathrm{T}} c / 8$. We introduce the subscript "pure" here to stress that this result is for a pure radiation fireball.

\section{BARYONIC LOADING}

The generalization of our current results to a loaded fireball is straightforward. For simplicity, let us consider a fireball loaded with some protons and their associated electrons. As discussed in Nakar et al. (2005), the electrons that accompany the protons contribute to the opacity, while the protons contribute to the inertia. Therefore, we can replace $m_{e}$ in equation (27) by the average mass per electron

$$
\bar{m}_{e}=\frac{2 N_{ \pm} m_{e}+N_{p} m_{p}}{2 N_{ \pm}+N_{p}}
$$

and obtain accurate results for a baryonic loaded fireball. Let us denote the mass-loading rate to be $\dot{M}_{p}$ and define $\eta=L / \dot{M}_{p} c^{2}$ to characterize a loaded fireball. There are three critical values of this ratio:

$$
\eta_{1}=\frac{L}{2 \dot{N}_{\infty} m_{e} c^{2}}=\frac{3}{16 \mathcal{N}_{\infty}\left(T_{ \pm}\right)}\left(\frac{L \sigma_{\mathrm{T}} T_{ \pm}^{3}}{4 \pi r_{i} m_{e} c^{3} T_{i}^{3}}\right)
$$

corresponds to a loaded fireball with equal mass for protons and pairs,

$$
\eta_{2}=\frac{L}{2 \dot{N}_{\infty} m_{p} c^{2}}=\frac{3}{16 \mathcal{N}_{\infty}\left(T_{ \pm}\right)}\left(\frac{L \sigma_{\mathrm{T}} T_{ \pm}^{3}}{4 \pi r_{i} m_{p} c^{3} T_{i}^{3}}\right)
$$

marks equal number densities of pairs and protons, and

$$
\eta_{3}=\left(\frac{m_{e}}{m_{p}}\right)^{1 / 4} \gamma_{\infty, \text { pure }}=1.03\left(\frac{L \sigma_{\mathrm{T}}}{4 \pi m_{p} c^{3} r_{i}}\right)^{1 / 4}
$$

is defined by the condition that the photons have effectively transferred all their energy to the protons (eq. [27]). Here we have improved the results of Nakar et al. (2005) by adding accurate factors before the expressions in the parentheses. The scaling relations in equation (27) have already been obtained by Nakar et al. (2005), so we can modify their equations (17)-(18) to include our numerical accurate factors here and obtain

$$
\gamma_{\infty, \text { load }}= \begin{cases}\gamma_{\infty, \text { pure }}, & \eta_{1} \ll \eta, \\ \left(\eta / \eta_{1}\right)^{1 / 4} \gamma_{\infty, \text { pure }}, & \eta_{2} \ll \eta \ll \eta_{1}, \\ \eta_{3}, & \eta_{3} \ll \eta \ll \eta_{2}, \\ \eta, & \eta \ll \eta_{3} .\end{cases}
$$

The energy that remains in the ejecta is

$$
L_{\infty, \text { load }}= \begin{cases}L_{\infty, \text { pure }}, & \eta_{1} \ll \eta, \\ \left(\eta / \eta_{1}\right)^{-3 / 4} L_{\infty, \text { pure }}, & \eta_{2} \ll \eta \ll \eta_{1}, \\ \eta_{3} / \eta L, & \eta_{3} \ll \eta \ll \eta_{2}, \\ L, & \eta \ll \eta_{3},\end{cases}
$$

where $L_{\infty, \text { pure }}=2 m_{e} c^{2} \dot{N}_{\infty, \text { pure }} \gamma_{\infty \text {, pure }}$ and $L$ is the photon luminosity. Here we note that we can use $\bar{m}_{e}$ defined in equation (28) to obtain accurate results near the transition point $\eta \sim \eta_{1}$. When $\eta \ll \eta_{2}$, the number of electrons that are associated with baryons exceeds the number of pairs, our calculation of the surviving number of pairs is no longer valid, since the excess amount of electrons 


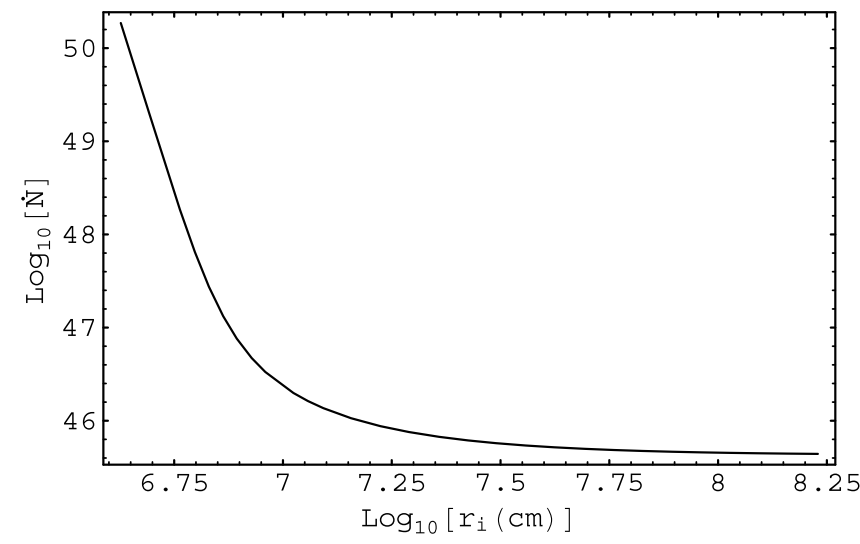

FIG. 3.-Number of pairs per unit time in SGRs, the values of parameters are $r_{i}=10^{6} \mathrm{~cm}$ and $L=10^{47} \mathrm{erg} \mathrm{s}^{-1}$. We have taken the logarithm (to base 10) of the horizontal and vertical axes.

will more easily annihilate positrons. The number of surviving positrons will be smaller than our estimate for $\dot{N}_{\infty}$ (eq. [19]). However, this has no energetic consequence, since in this case the energy is carried by the baryons instead of the pairs.

\section{APPLICATION TO SGRs}

We have numerically solved equations (16) and (21) for typical values of parameters for SGRs. In Figure 3 we plot the number of pairs per unit time in SGRs. Clearly, the electron-positron pairs freeze out. In Figure 4 we plot the evolution of the electron Lorentz factor. This figure shows that the Lorentz factor of electron-positron pairs increases and reaches its asymptotic value.

Let us compare these numerical results with their former estimates (Grimsrud \& Wasserman 1998; Nakar et al. 2005). Consider the situation of a typical SGR where an energy $E$ is deposited in the vicinity of a neutron star within the duration time $t$. In this case, the number of electrons emitted per unit time is

$$
\begin{aligned}
\dot{N}_{\infty} & =4 \pi r^{2} n_{e} \gamma_{e} c \\
& \approx 1.95 \frac{4 \pi r_{i} c^{2}}{\left\langle\sigma_{\mathrm{ann}} v\right\rangle}\left(\frac{T_{i}}{T_{ \pm}}\right)^{3} \approx 1.8 \times 10^{45} L_{47}^{3 / 4} r_{i, 6}^{-1 / 2}
\end{aligned}
$$

where the luminosity $L=16 \pi r_{i}^{2} a c T_{i}^{4} / 3$ is measured in units of $10^{47} \mathrm{erg} \mathrm{s}^{-1}$ and $r_{i}$ in units of $10^{6} \mathrm{~cm}$. For comparison, equation (61) of Grimsrud \& Wasserman (1998) gives the emission rate of the pairs as $\dot{N}_{\infty} \approx 4 \pi r_{i} c^{2} T_{i}^{3} /\left\langle\sigma_{\text {ann }} v\right\rangle T_{ \pm}^{3}$, which is about half of our estimate. ${ }^{1}$

Based on the photon luminosity $L=(16 \pi / 3) c r_{i}^{2} a T_{i}^{4}$, we obtain the initial temperature to be

$$
T_{i}=195 \mathrm{keV} L_{47}^{1 / 4} r_{i}^{-1 / 2}
$$

This estimate agrees very well with the observation (Hurley et al. 2005). In addition, the giant flare from SGR 1806-20 has an optically thin thermal spectrum (Hurley et al. 2005). From this ev-

\footnotetext{
1 Note the small differences between the definitions of $T_{ \pm}$of our eq. (13) and eq. (54) of Grimsrud \& Wasserman (1998). We have also defined our initial radius $r_{i}$ such that the initial Lorentz factor $\gamma_{i}=1$. We have taken these small differences into account when comparing with their results.
}

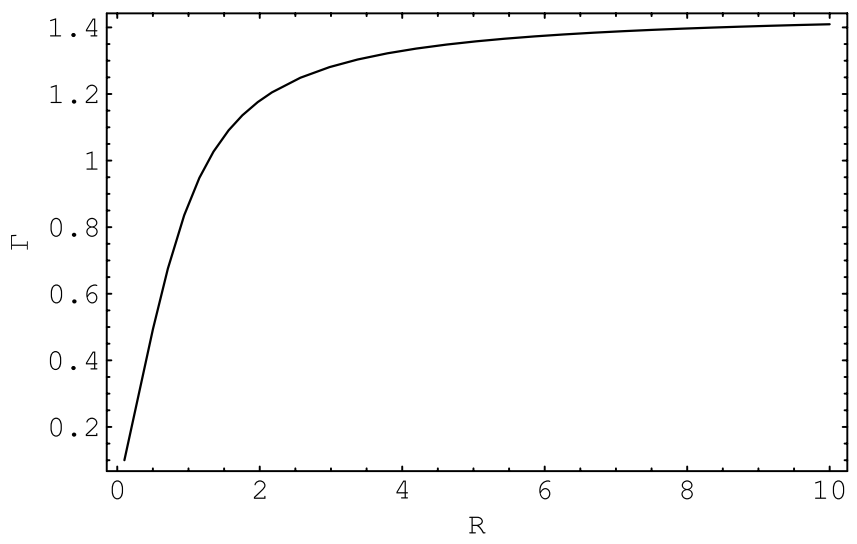

FIG. 4.- Normalized electron Lorentz factor $\Gamma$ defined in eq. (24). Here we plot the numerical solution to the dimensionless eq. (25).

idence it is reasonable to conjecture that we have directly observed the photons from the fireball. This is different from the usual GRBs, where we observe the nonthermal photons from the shocks between the fireball and the environment.

From equation (26), we find that the coasting Lorentz factor is (assuming $\sigma_{\text {tot }} \approx \sigma_{\mathrm{T}}$, the Thomson cross section)

$$
\gamma_{\infty} \approx 7.0 \times 10^{2} L_{47}^{1 / 4} r_{i, 6}^{-1 / 4}
$$

In equation (71) of Grimsrud \& Wasserman (1998), $\gamma_{\infty} \approx 5.3 \times$ $10^{2} L_{47}^{1 / 4} r_{i, 6}^{-1 / 4}$, which is smaller than our value. This is because they only expanded the flux of photons to quadrupole terms (eqs. [36] and [66] of Grimsrud \& Wasserman 1998).

Finally, after combining equations (34) and (36), the total kinetic energy of electron-positron pairs is

$$
E_{\infty}=2 m_{e} c^{2} \dot{N}_{ \pm} t \gamma_{\infty} \approx 2.1 \times 10^{41} \operatorname{erg} E_{46} r_{i, 6}^{-3 / 4}
$$

This result is slightly larger than previous estimates (Grimsrud \& Wasserman 1998; Nakar et al. 2005). It is shown in Nakar et al. (2005) that the amount of kinetic energy in the pair outflow is too small to produce the observed radio flux. Our numerical results that arrive at a slightly higher pair energy cannot solve the energy budget problem either. It is still 2 orders of magnitude short of producing the observed radio afterglow. Thus, a pure electronpositron-photon fireball cannot explain SGR 1806-20. A viable explanation is that the fireball is loaded with baryons or an electromagnetic field. As we have shown above, the ejecta of a heavy baryonic loaded fireball can carry enough energy and explain the observations. Electromagnetic loading is also quite natural, since the magnetar model of SGRs assumes the central engine to be a neutron star with strong magnetic field (Thompson \& Duncan 1995). The radio afterglow produced by this loaded fireball is further discussed in Taylor \& Granot (2006), Granot \& Taylor (2005) and Wang et al. (2005).

We thank Ehud Nakar and Ben Collins for useful discussion and correcting earlier versions of this manuscript. C. L. is supported by a Betty and Gordon Moore Fellowship. R. S. is a Packard Fellow and an Alfred P. Sloan Fellow. This research was partially supported by a NASA ATP grant. 


\section{APPENDIX}

\section{THE ANALYTICAL SOLUTION OF EQUATION (16)}

Here we consider the limiting solution of equation (16). To simplify our expressions, we measure temperature by the electron mass, i.e., we convert the temperature by $T \rightarrow k_{\mathrm{B}} T / m_{e} c^{2}$. Therefore, the three constants $k_{\mathrm{B}}, m_{e}$, and $c$ will not appear in this section.

As $T_{ \pm}$decreases, the dimensionless radius where equilibrium is effectively broken gets closer and closer to unity. Therefore, we can divide the solution of equation (16) into two intervals, $\mathcal{R} \in\left[\mathcal{R}_{0}, 1\right]$ and $\mathcal{R} \in[1, \infty)$, where $\mathcal{R}_{0}$ is some fiducial radius where we set the initial condition to be $\mathcal{N}\left(T_{ \pm}, \mathcal{R}_{0}\right)=\mathcal{R}^{3 / 2} e^{-\left(\mathcal{R}_{0}-1\right) / T_{ \pm}}$. When $\mathcal{R} \in\left[\mathcal{R}_{0}, 1\right]$, we can approximate equation (16) by

$$
\frac{d \mathcal{N}}{d \mathcal{R}}=-\mathcal{N}^{2}+e^{-2(\mathcal{R}-1) / T_{ \pm}} .
$$

The solution to this equation has an analytical form

$$
\mathcal{N}\left(T_{ \pm}, \mathcal{R}\right)=\frac{e^{-(\mathcal{R}-1) / T_{ \pm}}\left[2 K_{1}\left(T_{ \pm} e^{-(\mathcal{R}-1) / T_{ \pm}}\right)-C_{0} I_{1}\left(T_{ \pm} e^{-(\mathcal{R}-1) / T_{ \pm}}\right)\right]}{2 K_{0}\left(T_{ \pm} e^{-(\mathcal{R}-1) / T_{ \pm}}\right)+C_{0} I_{0}\left(T_{ \pm} e^{-(\mathcal{R}-1) / T_{ \pm}}\right)}
$$

where $I_{0}, I_{1}, K_{0}$, and $K_{1}$ are the modified Bessel functions of the first and second kinds and $C_{0}$ is the integration constant to be determined by the initial condition.

When $x \ll 1$, we can use the following asymptotic forms of those modified Bessel functions (Arfken 2005)

$$
K_{1,0}(x) \gg I_{1,0}(x), \quad K_{1}(x)=\frac{1}{x}+O\left(x^{2}\right), \quad K_{0}(x)=-\gamma_{\mathrm{Eu}}+\ln 2-\ln x+O\left(x^{2}\right),
$$

where $\gamma_{\mathrm{Eu}}=0.577216 \ldots$ is the Euler constant. Plugging these relations into equation (A2), we obtain the analytical solution at $R=1$ according to

$$
\mathcal{N}\left(T_{ \pm}, 1\right)=\frac{1}{T_{ \pm}\left(-\gamma_{\mathrm{Eu}}+\ln 2-\ln T_{ \pm}\right)} .
$$

Given this as the initial condition, we can solve equation (16) in the interval $\mathcal{R} \in[1, \infty)$. This time we approximate equation (16) as

$$
\frac{d \mathcal{N}}{d \mathcal{R}}=-\frac{\mathcal{N}^{2}}{\mathcal{R}^{4}},
$$

which has the analytical solution

$$
\lim _{T_{ \pm} \rightarrow 0} \mathcal{N}\left(T_{ \pm}, \mathcal{R}\right)=\frac{-1}{\left(1 / 3 \mathcal{R}^{3}\right)+C_{1}},
$$

where $C_{1}$ is another integration constant determined by the initial condition equation (A4). Substituting the initial condition equation (A4), we obtain the analytical expression for the number of freezeout pairs according to

$$
\mathcal{N}_{\text {ana }}=\frac{3}{1+\left(\ln 8-3 \gamma_{\text {Eu }}\right) T_{ \pm}-3 T_{ \pm} \ln T_{ \pm}} .
$$

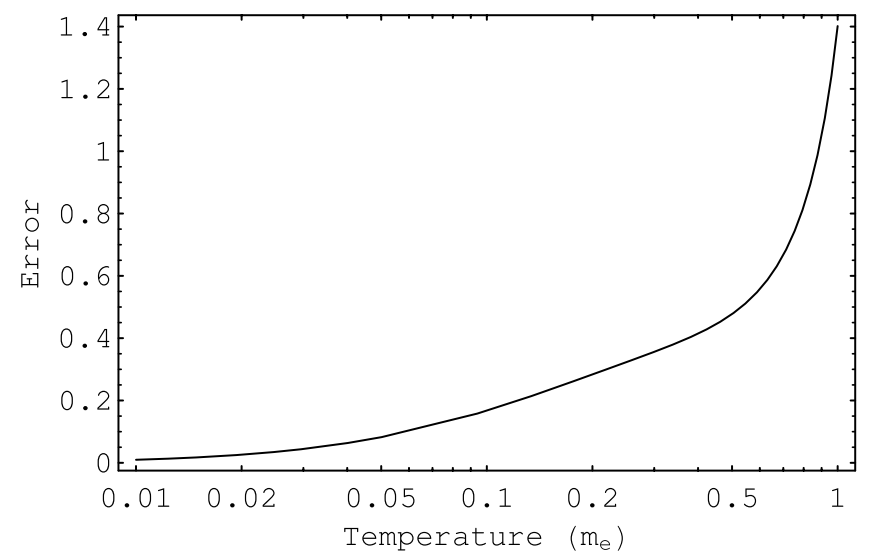

FIG. 5.- Relative error of our analytical solution compared to the numerical solution. As the equilibrium temperature $T_{ \pm}$decreases, our analytical formula eq. (A7) becomes closer to the numerical exact solution. 
In Figure 5 we plot the relative error

$$
\Delta \equiv \frac{\mathcal{N}_{\text {ana }}-\mathcal{N}_{\text {num }}}{\mathcal{N}_{\text {num }}},
$$

where $\mathcal{N}_{\text {ana }}$ is the number of freezeout pairs obtained via direct numerical integration and $\mathcal{N}_{\text {ana }}$ is the number of freezeout pairs obtained by the analytical method here. From Figure 5 we see that as $T_{ \pm}$decreases, our analytical formula becomes closer to the numerical solution. For example, when $T_{ \pm} \leq 0.1 m_{e}$, our analytical formula equation (A7) only introduces an error which is smaller than $17 \%$.

\section{REFERENCES}

Arfken, G. B. 2005, Mathematical Methods for Physicists (Burlington: Academic) Paczynski, B. 1986, ApJ, 308, L43

Goodman, J. 1986, ApJ, 308, L47

Granot, J., \& Taylor, G. B. 2005, ApJ, 625, 263

Grimsrud, O. M., \& Wasserman, I. 1998, MNRAS, 300, 1158

Hurley, K., et al. 2005, Nature, 434, 1098

Kouveliotou, C., et al. 1998, Nature, 393, 235

Mészáros, P., Laguna, P., \& Rees, M. J. 1993, ApJ, 415, 181

Nakar, E., Piran, T., \& Sari, R. 2005, ApJ, 635, 516

Piran, T., Shemi, A., \& Narayan, R. 1993, MNRAS, 263, 861

Shemi, A., \& Piran, T. 1990, ApJ, 365, L55

Svensson, R. 1982, ApJ, 258, 321

Taylor, G. B., \& Granot, J. 2006, Mod. Phys. Lett. A, 21, 2171

Thompson, C., \& Duncan, R. 1995, MNRAS, 275, 255

. 1996, ApJ, 473, 322

Wang, X., et al. 2005, ApJ, 623, L29 